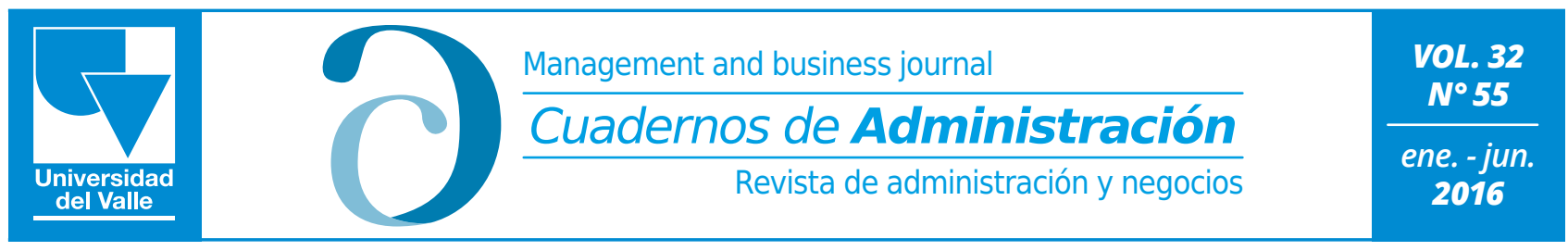

Print ISSN: 0120-4645 / E-ISSN: 2256-5078 / Short name: cuad.adm. / Pages: 47-58

Universidad del Valle / Facultad de Ciencias de la Administración / Universidad del Valle / Cali - Colombia

\title{
Alternativas de gestión del capital estructural en las instituciones de educación superior. Una propuesta para su evaluación
}

\author{
Alternatives of structural capital management in higher education institutions. A \\ proposal for their evaluation
}

Alternatives de Gestion du capital structurel dans les établissements d'enseignement supérieur. Une proposition pour l'évaluation

María del Rosario Demuner Floresa

Profesora investigadora, Coordinación de Investigación y Estudios de Posgrado, Facultad de Contaduría y Administración, Universidad Autónoma del Estado de México, México.

E-mail: mrdemunerf@uaemex.mx

Rosa María Nava Rogel ${ }^{b}$

Profesora investigadora, Coordinación de Investigación y Estudios de Posgrado, Facultad de Contaduría y Administración, Universidad Autónoma del Estado de México, México.

E-mail: rmnavar@uaemex.mx

Patricia Mercado Salgadoc

Profesora investigadora, Coordinación de Investigación y Estudios de Posgrado, Facultad de Contaduría y Administración, Universidad Autónoma del Estado de México, México.

E-mail: pmercados@uaemex.mx

Research article, PUBLINDEX-COLCIENCIAS clasification
Submmit: $09 / 11 / 2015$
Review: $02 / 05 / 2016$
Accepted: $15 / 06 / 2016$
Eje temático: Administración y Organizaciones

\section{Resumen}

El capital estructural es el conocimiento propio de la institución que surge en la medida en que es poseído por sus integrantes, explicitado, codificado y sistematizado formalmente; por ello el objetivo de este trabajo es identificar las dimensiones e indicadores que vislumbren la directriz de la gestión del capital estructural con el fin de hacer una propuesta de su evaluación que se use para mejorar el desempeño en las instituciones de educación superior. La revisión de 25 documentos teóricos y empíricos aporta dimensiones e indicadores coincidentes y redundantes en: 1) procesos: rutinas internas, sistematización de la información, innovación y nuevas tecnologías, dotación de equipo tecnológico; 2)

a Contador Público, Doctora en Ciencias Económico Administrativas, Universidad Autónoma del Estado de México, México.

b Contador Público, Instituto Tecnológico de Estudios Superiores de Monterrey, Doctora en Ciencias Económico Administrativas, Universidad Autónoma del Estado de México, México.

c Licenciada en Administración, Universidad Autónoma del Estado de México, Doctora en Administración, Universidad Nacional Autónoma de México, México. 
cultura: vínculos organizativos, aprendizaje organizativo, filosofía de la dirección, apoyo a la investigación, integración de mecanismos de coordinación; 3) estructura: integración de grupos de investigación internos, creación de bases de datos, publicación de revistas científicas; 4) propiedad intelectual: patentes y prototipos, publicaciones, conferencias impartidas, proyectos de investigación concluidos.

Palabras clave: capital estructural, evaluación, dimensiones, indicadores, instituciones de educación superior.

\begin{abstract}
Structural capital is the knowledge owned by an institution that arises in the extent that it is owned by its members, made explicit, codified and formally systematized; therefore the goal of this work is to identify the dimensions and indicators that glimpse the guideline of structural capital management in order to make a proposal for its evaluation that can be used to improve performance in higher education institutions. A review of 25 theoretical and empirical documents provides dimensions and overlapping and redundant indicators in: 1) processes: internal routines, systematization of information, innovation and new technologies, provision of technological equipment; 2) culture: organizational links, organizational learning, philosophy of management, research support, integration of coordination mechanisms; 3) structure: integration of internal research groups, databases creation, publication of scientific journals; 4) intellectual property: patents and prototypes, publications, lectures given, completed research projects.
\end{abstract}

Keywords: structural capital, evaluation, dimensions, indicators, higher education institutions.

\title{
Résumé
}

Le capital structurel est la connaissance qu'on a de l'institution qui se socialise dans la mesure où elle est détenue par ses membres, explicite, formellement codifié et systématisé ; I'objectif de ce travail et $d$ 'identifier les dimensions et les indicateurs entrevoyant la directrice sur la gestion du capital structurel afin de faire une proposition pour l'évaluation qui sera utilisée pour améliorer les performances dans les établissements d'enseignement supérieur. La révision de 25 documents théoriques et empiriques apporte des dimensions et des indicateurs coïncidants et redondants : 1) processus: routines internes, systématisation de l'information, innovation et nouvelles technologies, dotation d'équipe technologique 2) culture: liens organisationnels, apprentissage organisationnel, philosophie de la direction, soutien à la recherche, intégration de mécanismes de coordination 3) structure: intégration de groupes internes de recherche, création de bases de données, publication de revues scientifiques 4) propriété intellectuelle : brevets et prototypes, publications, conférences données, projets de recherche conclus.

Mots Clés: capital structurel, évaluation, dimensions, indicateurs, établissements d'enseignement supérieur.

\section{Introducción}

La economía del conocimiento, refiere el estudio de los procesos de creación, distribución y uso del conocimiento como el mayor impulsor de crecimiento, riqueza y empleo (Heng, Toh, Chin, Hsiu y Choo, et al., 2002). El conocimiento es el activo más importante de los bienes de capital y mano de obra porque su alcance y grado de sofisticación se permea en las actividades económicas y sociales. Según el World Bank (2006) el conocimiento debe ser uno de los pilares principales ubicados en el centro de la estrategia de crecimiento de cualquier país, su base es precisamente la educación, for- mación y capacitación nacional; es decir, la fuerza de trabajo debe estar integrada por trabajadores calificados capaces de actualizar y adaptar sus habilidades para crear conocimiento de forma eficiente.

Siendo el conocimiento la base del progreso y desarrollo económico de cualquier país, en virtud de la contribución de la productividad individual, de la agregación de valor que las personas son capaces de realizar mediante desarrollos tecnológicos, innovaciones en productos y servicios, procesos, etc. son las instituciones de educación superior (IES) quienes deben constituirse como fuente esencial de las oportu- 
nidades de formación (Rodríguez-Ponce y Palma-Quiroz, 2010). La educación superior es el determinante de la creación y difusión del conocimiento, de la formación y consolidación del capital humano avanzado.

Es así que la economía del conocimiento impone a los sistemas de educación superior el desafío de constituirse en un elemento básico para generar mayores niveles de competitividad tanto en las empresas como en el país.

Las IES, sus estructuras organizativas y la propia relevancia del sistema educativo como institución creadora y reproductora del conocimiento, son parte medular en el crecimiento económico porque este afecta tanto la oferta como la demanda de procesos de innovación porque una fuerza laboral y empresarial capacitada estará en mejor posición de crear, adoptar y transformar tecnologías, así como de generar una demanda de profesionales capaces de cubrir las expectativas de la misma comunidad estudiantil, de las organizaciones y de la sociedad en general.

Para que las IES logren encaminar sus actividades hacia la generación de un valor agregado en sus servicios y desde la perspectiva de las organizaciones, no solo deben enfocar sus esfuerzos a crear y difundir conocimiento, sino que también, internamente, deben capitalizar ese aprendizaje de sí mismas, diseñar y alinear estrategias de acuerdo con su misión de proveer a la sociedad educación de alta calidad e investigación pertinente.

Su problemática para lograr que la acumulación de conocimiento genere un valor adicional, radica además de la identificación de las capacidades y talentos de su personal, en la disposición que tengan para compartirlo, a la vez, también debe identificarse conocimiento en las relaciones con otros agentes y finalmente lograr una sistematización que permita una apropiación del conocimiento que redunde en innovaciones educativas. Para llevar a cabo una gestión eficiente del aprendizaje como parte del esfuerzo de toda la comunidad académica y administrativa, se requiere transmitir, compartir, comunicar y tangibilizar el conocimiento que se gesta en su interior (Bueno-CIC, 2003).
A partir de la necesidad de identificar y medir el conocimiento que aporta valor a las organizaciones, surge una corriente llamada gestión del conocimiento que mediante la realización de un conjunto de procesos y sistemas, aspectos tácticos, operacionales como creación, captura, transformación y uso busca incrementar significativamente el capital intelectual, aspectos intelectuales a partir de los recursos humanos y relaciones que constituyen el Know how de la organización (Euroforum Escorial, 1998).

En este sentido el capital intelectual juega un papel protagónico como gestor de riqueza en las organizaciones y en el país (Bradley, 1997), como fuente de creación de ventaja competitiva sostenida para la empresa (Bontis y Fitz-enz, 2002; Swart, 2006); ofrece un mayor rendimiento y creación de valor a las empresas (Holmen, 2005) e impulsa la competitividad (Davenport y Prusak, 2000) y el desarrollo regional.

El capital intelectual (CI) es aquel conocimiento que puede en el futuro ser convertido en beneficio, se encuentra formado por recursos tales como las ideas, los inventos, las tecnologías, los programas informáticos, los diseños y los procesos que promueven la capacidad de organización (Bontis, 1998) que operan en el presente y en el futuro (Stewart, 1998).

Bueno, Salmador y Merino (2008) después de realizar un estudio de los diferentes conceptos de capital intelectual lo definen como la

\section{acumulación de conocimiento que crea valor o riqueza cognitiva poseída por una organiza- ción, compuesta por un conjunto de activos de naturaleza intangible o recursos y capaci- dades basados en conocimiento, que cuando se ponen en acción, según determinada estra- tegia, en combinación con el capital físico o tangible, es capaz de generar ventajas com- petitivas o competencias esenciales para la organización en el mercado (p. 53).}

Dada la importancia que para cualquier organización tienen los intangibles, este trabajo se enfoca en el reconocimiento del capital intelectual como factor que permite la creación de valor a las IES, específicamente en el capital estructural (CE). Para ello, el objetivo que se persigue en este trabajo es identificar las dimensiones e indica- 
dores que vislumbren la directriz de la gestión del capital estructural con el fin de lograr un mejor desempeño en la institución, esto en virtud de ser el capital que denota el formalismo y sistematización del conocimiento y que a diferencia de los otros dos, está constituido por el conocimiento existente en la organización que es de su propiedad (IADE-CIC, 2003) ya que cuando las personas abandonan la organización este conocimiento permanece en ella (Euroforum Escorial, 1998).

A partir de una revisión conceptual y empírica, el trabajo se divide en una parte introductoria que describe la importancia del conocimiento en la economía actual; la segunda parte presenta aportaciones teóricas que definen y ubican el CE como dimensión del CI en IES; finalmente se presentan las aportaciones derivadas de la revisión de la literatura donde se contemplan las dimensiones e indicadores que permiten la evaluación del capital estructural.

\section{Capital estructural}

En el contexto universitario, el CI incluye todos los activos no tangibles o no físicos de la institución, como son: el conocimiento tácito de sus miembros, sus capacidades, talentos y destrezas, su red de colaboradores, vínculos externos, reconocimiento de la sociedad, sus procesos, capacidad de innovación, registro de patentes, entre otros. Estos activos han sido categorizados en tres capitales interrelacionados (Leitner, 2004; Ramírez, Lorduy y Rojas, 2007; Bezhani, 2010):

1. Capital humano. Conjunto de conocimientos explícitos y tácitos adquiridos por la comunidad universitaria, mediante procesos de educación, socialización y actualización propios de su actividad (Ramírez, 2007). Está representado por el conjunto de saberes desarrollados a partir de las experiencias, habilidades y competencias, apoyado en la capacitación y actualización académica, así como el trabajo en equipo (del Valle, 2002; Bueno, Morcillo, Rodríguez, Luque, Cervera y Rodríguez, 2002).

2. Capital estructural. Es el conocimiento explícito relativo a los procesos internos de difusión, comunicación y gestión del conocimiento científico y técnico en la universidad (Ramírez et al., 2007).
3. Capital relacional. Conjunto de relaciones con agentes económicos, políticos e institucionales de las que se genera conocimiento y valor agregado para la universidad; constituye los procesos de desarrollo y conservación de la vinculación de la universidad con instituciones económicas y políticas (Ramírez et al., 2007; redes de trabajo con alumnos y empresas, porque a través de los vínculos formados con ambos agentes, es posible exportar e importar conocimiento y la difusión del CI dentro y fuera de la propia institución.

Estos tres capitales se complementan sistémicamente con el fin de agregar valor a los activos basados en el conocimiento que han sido creados y que son identificados o existen en la organización, por un conjunto de actividades intangibles que ponen valor al "conocimiento en acción" de las personas y grupos (IADE-CIC, 2003).

Es así que en la universidad, la gestión del capital intelectual debe aportar decisiones que generen un cambio para lograr innovación, mejores tecnologías, una educación de calidad que responda a los desafíos de un entorno cultural y tecnológico, que sea capaz de adaptarse a las condiciones propicias para que los individuos y las organizaciones desarrollen habilidades en el aprendizaje. Por tanto, se debe enfocar al desarrollo de su capital humano por ser quien desempeña la labor esencial de docencia, investigación y gestión, a su capital estructural por ser el que integra el conocimiento sistematizado e institucionalizado y al capital relacional que permite el aprendizaje de la vinculación entre agentes internos y externos. Los beneficios no solo harán cumplir la misión de la universidad al satisfacer la demanda social, crear y transmitir conocimiento, sino que contribuirán a la competitividad del país.

Específicamente, el CE es el conocimiento propio de la organización que surge en la medida en que es poseído por las personas y sus equipos, explicitado, codificado, sistematizado e internalizado mediante un proceso formal que opera a través de la creación de rutinas (Bueno, 2001, p. 18) y de la eficacia y eficiencia interna (Euroforum Escorial, 1998).

El CE es reconocido como medio para rentabilizar y proyectar hacia el futuro la inteligencia, el talento y el trabajo de todos sus 
miembros como propuesta de valor de la entidad (Bueno-CIC, 2003). Se relaciona con el conocimiento que ha sido capturado e institucionalizado dentro de la estructura, el proceso y la cultura de una organización. Hace referencia a la tecnología de información, de producto, de proceso, de organización, de propiedad intelectual (Sukhdev y Kansal, 2011).

Es importante precisar que el CE, integra intangibles no infraestructura tangible como el equipamiento. Los activos de infraestructura que considera son metodologías y procesos que hacen posible el funcionamiento de la organización. A veces, este concepto provoca confusión al integrar en él, el equipamiento; un claro ejemplo se representa con las tecnologías de información (TI) que son parte de una innovación tecnológica que ejercen una notable influencia en los procesos de generación, producción, transmisión, difusión y organización de la información y el conocimiento (Kaplan y Norton, 1992) que produce la creación de valor (Barney, 1991), pero son solo un mecanismo de ayuda al capital intelectual; la parte intangible que se integra al capital estructural son los productos que se generan con la ayuda de las TI, como pueden ser: diseño de software, procedimientos, bases de datos, sistemas de comunicación, etc.

En atención al Modelo Nova (Camisón, Palacios y Devece, 2000) creado por la empresa Nova Care y desarrollado por la comunidad Club de Gestión del Conocimiento de la Comunidad Valenciana, no refiere el capital estructural como tal, pero si considera el capital de organización como aquel que abarca los activos de conocimientos sistematizados, explícitos o interiorizados por la organización, tal como las ideas explícitas objeto de propiedad intelectual (patentes, marcas); conocimientos materializables en activos de infraestructura susceptibles de transmitirse y compartirse por varias personas como la descripción de invenciones, fórmulas, sistema de información y comunicación, tecnologías disponibles, documentación de procesos de trabajo, sistemas de gestión, estándares de calidad; conocimientos interiorizados compartidos en el seno de la organización de modo informal como las formas de hacer de la organización, rutinas, cultura (Camisón, Palacios y Devece, 2000, citado por González y Rodríguez, 2010).

\section{Metodología}

Edvinsson y Malone (1997) sugieren que la gestión del CI debe tener un objetivo muy claro, respecto a para qué se quiere medir este intangible; en este caso, el enfoque es hacia el $\mathrm{CE}$, que con una adecuada gestión se pretende mejorar el desempeño del CI.

En este sentido el objetivo que se persigue es identificar y definir las dimensiones e indicadores que intervienen en el diagnóstico del capital estructural en IES. Se busca proponer indicadores de capital estructural con la finalidad de que al ser empleados en las IES, se logre homogeneizar su aplicación práctica de tal suerte que en el futuro obtengan comparaciones respecto a la eficiencia que han logrado.

La información obtenida de las dimensiones e indicadores deberá cubrir el propósito de aportar información dirigida a los directivos y gestores de la institución e identificar en dónde reside su valor. De tal forma que la información que se obtenga permitirá hacer comparaciones más significativas teniendo en cuenta las metas previstas, su propia evolución en un determinado tiempo o resultados de otras IES cuyo uso producirá un mejor el desempeño en las IES.

Para ello, las dimensiones se consideran conjuntos de indicadores que permiten su clasificación. Y los indicadores, en general, permiten representar, en alguna medida, la dimensión teórica de una variable práctica. Específicamente, los indicadores de medición del capital estructural valoran los activos intangibles de las organizaciones expresados en unidades de medida (CIC, 2003).

Para el análisis de las dimensiones e indicadores del capital estructural se utilizó un proceso deductivo que, en primer lugar aportara las dimensiones estudiadas y luego el conjunto de indicadores para su identificación y medición. Este análisis se efectuó en dos fases: la primera corresponde a la detección de la literatura teniendo en cuenta artículos especializados redundantes en el tema. En la segunda fase, se utilizó una hoja de cálculo en las que se clasificaron los indicadores por afinidad y autor y se ponderó su peso.

Es así que mediante un estudio sistemático de 25 artículos de estudiosos prestigiados en 
el ramo que refieren el $\mathrm{CE}$, se hace un análisis para identificar las dimensiones e indicadores empleados. Con la detección de las dimensiones, se profundiza en la comprensión de los indicadores empleados de acuerdo con una serie de pautas comunes, materializadas en unos atributos y características básicas (Bueno et al., 2002). Una vez identificadas las dimensiones y los indicadores se realiza una descripción del uso de los mismos, con el enfoque de uso hacia la mejora de la gestión del capital estructural.

\section{Resultados}

\subsection{Dimensiones del capital estructural}

Las dimensiones que se han empleado en estudios empíricos tratan de identificar cómo el CE ha sido capturado e institucionalizado dentro de la estructura, los procesos internos, la cultura de una organización, formas de comunicación que permiten una eficiente y efectiva administración, y transmisión del conocimiento (Edvinsson y Malone, 1997; Sukhdev y Kansal, 2011). Son parte de él, las patentes, marcas y todo lo demás que sostiene la productividad (Edvinsson y Malone, 1997). Reconoce el conocimiento encajado en las rutinas organizativas (Crossan, Lane y White, 1999) y en la estructura por ser la parte facilitadora del desarrollo de las capacidades internas en nuevas y flexibles formas de competir, resolver problemas estructurales y facilitar el diseño de las estrategias (Bontis, 1998); los sistemas de información y comunicación, la tecnología disponible, los procesos de trabajo, los sistemas de gestión (Azofra, Prieto y Santidrian, 2001), las bases de datos (Youndt y Snell, 2004).

En la búsqueda de las dimensiones que conforman el CE, una aportación que ha sido base de clasificación para estudios posteriores, es la de Bueno-CIC (2003) quienes proponen el capital organizativo y el capital tecnológico como una división legitimada por sus diferentes implicaciones para la gestión. El primero asociado con el ámbito estructural de los diseños, procesos y cultura, integra todos los aspectos relacionados con la teoría administrativa, se refiere al diseño estructural, mecanismos de coordinación, comportamiento grupal, rutinas organizativas, cultura corporativa, sistemas de pla- neación y control, puede considerarse de carácter tácito. Y el segundo vinculado con el esfuerzo en investigación y desarrollo el uso de la dotación tecnológica y los resultados, tiene más bien un carácter explícito puesto de manifiesto por el conjunto de conocimientos técnicos como los recursos tecnológicos disponibles en la universidad, tales como recursos bibliográficos, documentales, archivos, desarrollos técnicos, patentes, licencias, software, bases de datos, etc.

El análisis realizado resume la coincidencia de cuatro dimensiones estudiadas:

1. Procesos. Hacen referencia a aquellos activos que otorgan valor por la forma en que se desarrollan dentro de ella y que aportan orden, seguridad, corrección y calidad a la organización y al contexto en el que los empleados se desenvuelven, como es el caso de operaciones, flujos de información, métodos, diagramas de flujo, procedimientos, metodologías que hacen posible el funcionamiento de la organización (Brooking, 1996; Kaplan y Norton, 1997; Ross, Ross, Edvinsson, y Dragonneti, 1997; Edvinsson y Malone, 1998; Bontis, 1998; Euroforum Escorial, 1998; Bueno-CIC, 2003; CIC, 2003; Chen, Zhu y Xie, 2004; Joia, 2004; Guthrie, Petty, Yongvanich y Ricceri, 2004; Ordóñez de Pablos, 2004; Topete y Bustos, 2007; Aguilera, Díaz y Hernández, 2011; Ramírez y Peñalver, 2013).

2. Cultura. Conjunto de normas, hábitos y valores que son compartidos por las personas y/o grupos que dan forma a una institución, la planeación estratégica y operacional, la normativa, la comunicación efectiva que es asumida por quien responde por los resultados operativos (Brooking, 1996; Saint-Onge, 1996; Sveby, 1997; Bontis, 1998; Euroforum Escorial, 1998; Bontis, 1998; Dow, 1998; CIC, 2003; Bueno-CIC, 2003; Ordóñez de Pablos, 2004; Chen et al., 2004; Joia, 2004; Guthrie et al., 2004).

3. Estructura. Se refiere al equilibrio organizativo, a la evaluación de los aspectos clave que mantienen y cohesionan la estructura de la organización y permite que las actividades se desarrollen de manera sistémica y coherente; se trata de la instrumentación de funcionamiento de la 
organización en su conjunto, por departamentos, áreas, filiales, unidades, según se configure la estructura organizativa (Saint-Onge, 1996; Sveby, 1997; Ross, et al., 1997; Edvinsson y Malone, 1997; Euroforum Escorial, 1998; Bontis, 1998; Bueno-CIC, 2003; CIC, 2003; Chen et al., 2004; Joia, 2004; Ordóñez de Pablos, 2004; Sánchez, Elena y Castrillo, 2009).

4. Propiedad intelectual. Hace referencia a cualquier aspecto de la organización que pueda generar valor futuro a través de patentes registradas y las ideas se derivan de la protección legal que la organización ejercita sobre aquellos activos que tienen un valor especial para la misma, entre los que se encuentran el know-how, los secretos de fabricación, el copyright, las patentes, los derechos de diseños y marcas de fábrica y servicios (Brooking, 1996; Sveby, 1997; Ross et al., 1997; Edvinsson y Malone, 1997; Edvinsson y Malone, 1998; Euroforum Escorial, 1998; CIC, 2003; Bueno-CIC, 2003; Guthrie et al., 2004; Topete y Bustos, 2007; Sánchez et al., 2009; Ramírez y Peñalver, 2013).

\subsection{Indicadores de capital estructural}

Un sistema de indicadores debe satisfacer plenamente las funciones de información y gestión cuando se concibe como un

De las dimensiones detectadas se realiza un análisis que desprende una serie de indicadores empleados (Tabla 1).

El diseño de un sistema de indicadores, debe considerar el cumplimiento de ciertos atributos, como los que se describen en la Tabla 2 .

Adicionalmente, los indicadores se definen a partir de algunos criterios, tomando en cuenta lo que se desea medir (Tabla 3).

Del análisis de los indicadores señalados en los artículos revisados y adaptados a IES, se mencionan los presentados en la Tabla 4; se presentan como valores absolutos que dependiendo del fin que se desee alcanzar, podrán expresarse en porcentaje o tasas de variación.

Si bien existe un sinnúmero de indicadores pueden reflejar la gestión del capital estructural de una institución educativa; existe el inconveniente en la obtención y estandarización de la información; es por esto que los indicadores anteriores han sido elegidos en función de la información estandarizada y publicada por una IES. Solo como ejemplo, su uso debe complementarse con aquellos que fortalezcan las dimensiones antes descritas, que respeten los atributos y criterios propuestos.

\section{Conclusiones}

La importancia de la evaluación del CE en la organización hace analizar y fortalecer el conocimiento que ha sido capturado y que debe institucionalizarse porque se encuentra encajado en los procesos internos, la cultura, estructura y propiedad intelectual que radica en la organización, por ser estas las dimensiones redundantes en las aportaciones de la literatura del tema.

Resaltan los indicadores para procesos: las rutinas internas, sistematización de la información, innovación y nuevas tecnologías, dotación de equipo tecnológico; para cultura: vínculos organizativos, aprendizaje organizativo, filosofía de la dirección, apoyo a la investigación; estructura: integración de mecanismos de coordinación, integración de grupos de investigación internos, creación de bases de datos, publicación de revistas científicas; propiedad intelectual: patentes, prototipos, publicaciones, conferencias impartidas, proyectos de investigación concluidos.

Al revisar las bases de datos consensados de las IES, se puede ver la falta de información que permite diagnosticar el CI. Además, cada institución presenta un informe anual, que al revisar los datos reportados se percibe que cada quien informa lo que más interesa a su institución, de tal forma que no existe una estandarización que permita hacer comparaciones entre ellas para medir el CE.

Ante la limitación de información, se cree que para que las IES puedan evaluar su CE con el fin de gestionarlo de mejor forma, deben acudir a la creación interna y sostenimiento de una serie de indicadores que realmente reflejen el status de las dimensiones que lo componen. Esto es también reforzar la cultura de autoevaluación. 
Tabla 1. Indicadores de capital estructural

\begin{tabular}{|c|c|c|}
\hline Dimensiones & Indicadores & Fuente \\
\hline \multirow[t]{4}{*}{ Procesos } & Rutinas internas & $\begin{array}{l}\text { Kaplan y Norton, 1992; Brooking, 1996; Kaplan y Norton, 1997; Edvinsson et al., } \\
\text { 1997; Euroforum Escorial, 1998; Edvinsson y Malone, 1997; Bontis, 1998; } \\
\text { Leitner, 2002; CIC, 2003; Bueno-CIC, 2003; Chen et al., 2004; Joia, 2004; } \\
\text { Guthrie et al., 2004; Ordóñez de Pablos, 2004; Topete y Bustos, 2007; } \\
\text { Aguilera, Díaz y Hernández, 2011; Ramírez y Peñalver, } 2013 .\end{array}$ \\
\hline & $\begin{array}{l}\text { Sistematización de } \\
\text { información }\end{array}$ & $\begin{array}{l}\text { Chen et al., 2004; Joia, 2004; Guthrie et al., 2004; Brooking, 1996; Saint-Onge, } \\
\text { 1996; Ross et al., 1997; Sveby, 1997; Edvinsson y Malone, 1998; Bontis, } \\
\text { 1998; Aguilera, Díaz y Hernández, 2011. }\end{array}$ \\
\hline & $\begin{array}{l}\text { Innovación y nuevas } \\
\text { tecnologías }\end{array}$ & $\begin{array}{l}\text { Bueno-CIC, 2003; CIC, 2003; Chen et al., 2004; Joia, 2004; Ordóñez de Pablos, } \\
\text { 2004; Ramírez y Peñalver, } 2013 .\end{array}$ \\
\hline & $\begin{array}{l}\text { Dotación de equipo } \\
\text { tecnológico }\end{array}$ & Brooking, 1996; Edvinsson y Malone, 1997; CIC, 2003; Bueno-CIC, 2003. \\
\hline \multirow[t]{5}{*}{ Cultura } & $\begin{array}{l}\text { Vínculos organizativos } \\
\text { internos }\end{array}$ & $\begin{array}{l}\text { Saint-Onge, 1996; Brooking, 1996; Sveby, 1997; Bontis, 1998; Euroforum } \\
\text { Escorial, 1998; Dow, 1998; Bueno-CIC, 2003; CIC, 2003; Chen et al., 2004; } \\
\text { Joia, 2004; Guthrie et al., 2004; Ordóñez de Pablos, 2004. }\end{array}$ \\
\hline & Aprendizaje organizativo & Leitner, 2002; Bueno-CIC, 2003; CIC, 2003; Chen et al., 2004. \\
\hline & Filosofía de la dirección & Brooking, 1996; Euroforum Escorial, 1998; Joia, 2004; Guthrie et al., 2004. \\
\hline & Apoyo a la investigación & Bueno-CIC, 2003; Bueno-CIC, 2003. \\
\hline & $\begin{array}{l}\text { Integración de mecanismos } \\
\text { de coordinación }\end{array}$ & $\begin{array}{l}\text { Saint-Onge, 1996; Sveby, 1997; Ross et al., 1997; Edvinsson y Malone, 1997; } \\
\text { Euroforum Escorial, 1998; Bontis, 1998; Bueno-CIC, 2003; CIC, 2003; Chen et } \\
\text { al., 2004; Joia, 2004; Ordóñez de Pablos, 2004; Sánchez et al., } 2009 .\end{array}$ \\
\hline \multirow[t]{4}{*}{ Estructura } & $\begin{array}{l}\text { Integración de grupos de } \\
\text { investigación internos }\end{array}$ & Carillo et al., 2012. \\
\hline & Creación de bases de datos & Brooking, 1996; Edvinsson y Malone, 1997; Joia, 2004. \\
\hline & $\begin{array}{l}\text { Acervo electrónico, } \\
\text { publicación de revistas } \\
\text { científicas }\end{array}$ & Bontis, 1998; Nava y Mercado, 2010; Fazlagic, 2011. \\
\hline & Patentes, prototipos & $\begin{array}{l}\text { Brooking, 1996; Edvinsson y Malone, 1997; Ross et al., 1997; Sveby, 1997; } \\
\text { Topete y Bustos, 2007; Euroforum Escorial, 1998; Modelo Navegador de } \\
\text { Skandia, Edvinsson y Malone, 1998; Bueno-CIC, 2003; CIC, 2003; Guthrie et } \\
\text { al., 2004; Sánchez et al., 2009; Ramírez y Peñalver, 2013. }\end{array}$ \\
\hline \multirow[t]{3}{*}{$\begin{array}{l}\text { Propiedad } \\
\text { intelectual }\end{array}$} & Publicaciones & $\begin{array}{l}\text { Topete y Bustos, 2007; Sánchez et al., 2009; Nava y Mercado, 2010; Aguilera, } \\
\text { Díaz y Hernández, 2011. }\end{array}$ \\
\hline & Conferencias impartidas & Topete y Bustos, 2007. \\
\hline & $\begin{array}{l}\text { Proyectos de investigación } \\
\text { concluidos }\end{array}$ & Aguilera et al., 2011; Naranjo-Herrera et al., 2013; Ramírez y Peñalver, 2013. \\
\hline
\end{tabular}


Tabla 2. Atributos de los indicadores de capital estructural

\begin{tabular}{l|l}
\hline \multicolumn{1}{c|}{ Atributo } & \multicolumn{1}{c}{ Conceptualización } \\
\hline Relevancia & Conserva el vínculo con el objetivo estratégico \\
\hline Correspondencia & Contribuye a desarrollar el proceso a medir \\
\hline Exactitud & Verifica lo que se desea medir \\
\hline Precisión & Confirma que los datos originales corresponden a la realidad y aporta ideas de adecuación del procedimiento \\
\hline Comparación & Guarda consistencia y estabilidad temporal \\
\hline \multicolumn{2}{c}{$\quad$ Fuente: Adaptado de CIC (2003). } \\
\hline
\end{tabular}

\begin{tabular}{l|l}
\hline \multicolumn{1}{c}{ Criterio } & \multicolumn{1}{c}{ Tabla 3. Criterios de indicadores de capital estructural } \\
\hline Dinámico & $\begin{array}{l}\text { Indicadores de actividad intangible: presentan una visión dinámica del CE, se enfocan a medir el proceso del } \\
\text { conocimiento en acción. } \\
\text { Indicadores de activo intangible: proporcionan una visión estática del intangible en un momento determinado. }\end{array}$ \\
\hline Funcional & $\begin{array}{l}\text { Indicadores de eficiencia: valoran la relación entre los resultados de una actividad y el desarrollo del intangible } \\
\text { empleado. } \\
\text { Indicadores de eficacia: miden el grado o nivel de consecución de los objetivos previstos. }\end{array}$ \\
\hline $\begin{array}{l}\text { Nivel de } \\
\text { información }\end{array}$ & $\begin{array}{l}\text { Indicadores de primer nivel: refieren al contexto en la organización. } \\
\text { Indicadores de segundo nivel: dan una idea global del potencial de la organización, expresan el valor del } \\
\text { intangible relacionando dos o más indicadores de primer nivel. }\end{array}$ \\
\hline $\begin{array}{l}\text { Escala de } \\
\text { medición }\end{array}$ & $\begin{array}{l}\text { Valores absolutos: expresan posicionamiento. } \\
\text { Valores relativos: valores porcentuales, tasas de variación. Su uso se enfoca a comparaciones entre variables, } \\
\text { respecto a las metas previstas, comparaciones entre instituciones similares, estudios longitudinales. }\end{array}$ \\
\hline & \multicolumn{1}{c}{ Fuente: Adaptado de CIC, 2003. } \\
\hline
\end{tabular}

Fuente: Adaptado de CIC, 2003.

\begin{tabular}{l|l|l}
\hline \multicolumn{2}{c}{ Tabla 4. Medición de indicadores } \\
\hline Dimensiones & \multicolumn{1}{c}{ Indicadores } & \multicolumn{1}{c}{ Medición } \\
\hline \multirow{2}{*}{ Procesos } & Rutinas internas & Número de procesos administrativos certificados \\
\cline { 2 - 3 } & Sistematización de información & Número de procesos administrativos automatizados \\
\cline { 2 - 3 } & Innovación y nuevas tecnologías & $\begin{array}{l}\text { Número de software de simulación y paquetería para apoyo } \\
\text { administrativo adquiridos }\end{array}$ \\
\cline { 2 - 3 } & Dotación de equipo tecnológico & Número de equipos dotados en el período de estudio \\
\hline \multirow{2}{*}{ Cultura } & Vínculos organizativos internos & $\begin{array}{l}\text { Número de áreas o departamentos sin observaciones en } \\
\text { auditorías }\end{array}$ \\
\cline { 2 - 3 } & Aprendizaje organizativo & $\begin{array}{l}\text { Número de cursos didácticos o de especialización tomados } \\
\text { por profesores y personal administrativo }\end{array}$ \\
\cline { 2 - 3 } & Filosofía de la dirección & Número de metas cumplidas de planes estratégicos \\
\cline { 2 - 3 } & Apoyo a la investigación & $\begin{array}{l}\text { Número de apoyos otorgados a profesores en congresos, } \\
\text { publicaciones, proyectos }\end{array}$ \\
\hline Estructura & Integración de mecanismos de coordinación & $\begin{array}{l}\text { Número de reuniones de academia } \\
\text { Número de programas de estudio revisados y aprobados }\end{array}$ \\
\cline { 2 - 3 } & Integración de grupos de investigación internos & Número de grupos de investigación \\
\cline { 2 - 3 } & Creación de bases de datos & Número de bases de datos creadas \\
\cline { 2 - 3 } & Publicación de revistas científicas & Número de revistas para publicar \\
\cline { 2 - 3 } & Acervo electrónico & Número de textos y revistas digitales de consulta \\
\hline Propiedad & Patentes, prototipos & Número de patentes o prototipos registrados \\
\cline { 2 - 3 } intelectual & Publicaciones & Número de artículos científicos publicados \\
\cline { 2 - 3 } & Conferencias impartidas & Número de proyectos de investigación concluidos \\
\hline & Proyectos de investigación concluidos & Fuente: Adaptado de CIC, 2003. \\
\hline & & \\
\hline
\end{tabular}




\section{Referencias}

Aguilera, C. S., Díaz, C. M., y Hernández, C. Y. (2011). El capital intelectual en la gestión del conocimiento de las universidades. Cuadernos de Educación y Desarrollo, 3(24). Recuperado dehttp://www.eumed.net/rev/ced/24/ccc.htm

Azofra, V., Prieto, B., y Santidrian, A. (2001). Los indicadores de rendimiento y su contribución a la creación, medición y valoración de capital intelectual. Estudio de caso: Nuevas tendencias en dirección de empresas (Documento de Trabajo 16/01). Burgos, España: Universidad de Burgos, Universidad de Salamanca, Universidad de Valladolid.

Barney, J. (1991). Firm resources and sustained competitive advantage. Journal of Management, 17(1), 99-120.

Bezhani, I. (2010). Intellectual capital reporting at UK universities. Journal of Intellectual Capital, 11(2), 179-207.

Bontis, N. (1998). Intellectual capital: an exploratory study that develops measures and models. Management Decision, 36(2), 63-76.

Bontis, N., \& Fitz-enz, J. (2002). Intellectual capital ROI: a causal map of human capital antecedents and consequents. Journal of Intellectual Capital, 3(3), 223-247.

Bradley, K. (1997). Intellectual capital and the new wealth of nations. Business Strategy Review, 8(1), 53-62.

Brooking, A. (1996). El capital intelectual. El principal activo de las empresas del tercer milenio. Barcelona, España: Ediciones Paidós Ibérica S. A.

Bueno, C. E. (1998). El capital intangible como clave estratégica en la competencia actual, Boletín de Estudios Económicos, LIII(164), $207-$ 229.

Bueno, C. E. (2001). El pensamiento integrado. Retos y claves para la dirección de empresas en el nuevo milenio. Dirección y Progreso, 179, 26-31.

Bueno C. E. (2002) La sociedad del conocimiento: Creación, medición y gestión del conocimiento en las organizaciones. Madrid, España: IADE-CIC.

Bueno, C. E., y CIC. (2003). Modelo Intellectus: Medición y Gestión del Capital Intelectual. (Documento Intellectus, 5). Madrid, España: CIC-IADE (UAM).

Bueno, E., Morcillo, P., Rodríguez, J., Luque, M. A., Cervera, M., y Rodríguez, O. (2002). Indicadores de Capital Intelectual aplicados a la Ac- tividad Investigadora y de Gestión del conocimiento en las Universidades y Centros Públicos de Investigación de la Comunidad de Madrid. En A. Modrego (Ed.), Capital intelectual y producción científica. Madrid, España: Dirección General de Investigación, Consejería de Educación, Comunidad de Madrid.

Bueno, C. E., Salmador, M. P., y Merino, C. (2008). Génesis, concepto y desarrollo del capital intelectual en la economía del conocimiento: Una reflexión sobre el Modelo Intellectus y sus aplicaciones. Estudios de Economía Aplicada, 26,(2), 43-63.

Bueno, E. (2003). Modelo Intellectus: Medición y gestión del capital intelectual. Madrid, España: DICREA Creaciones Gráficas.

Camisón, C., Palacios, D., y Devece, C. (2000). Un nuevo modelo para la medición del Capital Intelectual: El modelo NOVA. Ponencia presentada en el X Congreso Nacional de Asociación Científica de Economía y Dirección de la Empresa, (OCEDE), Oviedo, España.

Carillo, Z. E., Gutiérrez P. F., y Díaz, S. C. A. (2012). Propuesta de indicadores para gestión del capital estructural en grupos de investigación. Universidad \& Empresa, (22), 99-130.

Chen, J., Zhu, Z., \& Xie, Y. (2004). Measuring intellectual capital: a new model and empirical study. Journal of Intellectual Capital, 5, 195-212.

Centro de Investigación sobre la Sociedad del Conocimiento (CIC). (2003). Modelo Intellectus: Medición y Gestión del Capital Intelectual (Intellectus $\mathrm{N}^{\circ}$ 5). Madrid, España: CIC

Crossan, M. M., Lane, H.W., \& White, R. E. (1999). An Organizational Learning Framework: From Intuition to Institution. Academy of Management Journal, 24, 522-537.

Davenport, T. H., \& Prusak, L. (2000). Working knowledge: How organizations manage what they know. Boston, USA: Harvard Business School Press.

del Valle, R. (2002). Diseño de un modelo de gestión del conocimiento para la UNELLEZ que promueva el desarrollo de ventajas competitivas en el área de investigación. Barinas, Venezuela: Universidad Nacional Experimental de los Llanos Occidentales Ezequiel Zamora.

Dow CHemical. (1998). Model of dow chemical. Recuperado de http://bvs.sld.cu/revistas/aci/ vol13_6_05/aci060605.h tm

Edvinsson, L., \& Malone, M. (1997). Intellectual capital: Realizing your company's true value by 
finding its hidden brainpower. Nueva York, USA: Harper Collins Publishers.

Edvinsson, L., Roos, J., Roos, G., \& Dragonetti, N. C. (1997). Intellectual Capital: Navigating in the new business landscape. New York, USA: NYU Press.

Edvinsson, L., y Malone, M. (1998). El capital intelectual, cómo identificar y calcular el valor inexplorado de los recursos intangibles de su empresa. Bogotá, Colombia: Grupo Editorial Norma.

Euroforum Escorial. (1998). Medición del Capital Intelectual. Madrid, España: IUEE

Fazlagic, A. (2011, August). Measuring the intelectual capital of a university. OECD. Conference on Trends in the management of human resources in the higher education, OECD and IMHE, The Poznan University of Economics, Poznan, Poland.

González, M. J. J., y Rodríguez, D. M. (2010). Modelos de Capital Intelectual y sus indicadores en la universidad pública. Cuadernos de Administración, 26(43), 113-128.

Guthrie, J., Petty, R., Yongvanich, K., \& Ricceri, F. (2004). Using content analysis as a research method to inquire into intellectual capital reporting. Journal of Intellectual Capital, 5(2), 282-293.

Holmen, J. (2005). Intellectual Capital Reporting. Management Accounting Quarterly, 6(4), 1-9.

Instituto Universitario de Investigación en Administración del Conocimiento e Innovación de Empresas (IADE), Centro de Investigación sobre la Sociedad del Conocimiento (CIC). (2003). Modelo intellectus, medición y gestión del capital intelectual. (Documento Intellectus 5). Madrid, España: Universidad Autónoma de Madrid.

Joia, L. A. (2004). Are frequent customers always a company's intangible asset? Some findings drawn from an exploratory case study. Journal of Intellectual Capital, 5(4), 586-601.

Kaplan, R. S., \& Norton, D. P. (1996). The balanced scorecard: translating strategy into action. Boston, USA: Harvard Business Press.

Kaplan, R., \& Norton, D. P. (1992). The Balanced Scorecard -Measures That Drive Performance. Harvard Business Review, 70(1), 71-79.

Leitner K. H. (2002, November). Intellectual Capital reporting for Universities: Conceptual Background and Applications within the Recognition of Austrian universities. Conference The Trans- parent Enterprise. The Value of Intangibles, Autonomous University of Madrid Ministry of Economy, Madrid, Spain. http://www.academia. edu/3586272/Intellectual_Capital_Reporting for_Universities_Conceptual_background_and application_within the reorganisation_of_Austrian_universities

Leitner, K. H. (2004). Intellectual capital reporting for universities: conceptual background and applications for Austian universities. Research evaluations, 13(2), 129-140.

Naranjo-Herrera, C. G., Rubio-Jaramillo, J., Salazar-Mesa, L. M., Robledo-Martínez, A. V., y Duque-Trujillo, J. (2013). Indicadores de capital intelectual. Memorias, 11(19), 39-51.

Nava, R. R. M., y Mercado, S. P. (2010). Evaluación de la calidad métrica para indicadores de capital intelectual generados a partir de bases estadísticas. Revista de la Educación Superior, XXXIX(3), (155), 99-120.

Ordoñez de Pablos, P. (2004). Las cuentas de capital intelectual como complemento del informe anual. Economía Industrial, (357), 63-74.

Ramírez, C. Y., y Peñalver, S. J. (2013). Propuesta de un Informe de Capital Intelectual para las instituciones de educación superior españolas. Estudios de Economía Aplicada, 31(2), 525-554.

Ramírez, C. Y., Lorduy, C., \& Rojas, J. A. (2007). Intellectual capital management in Spanish universities. Journal of Intellectual Capital, 8(4), 732-748.

Rodríguez-Ponce, E., y Palma-Quiroz, A. (2010). Desafíos de la educación superior en la economía del conocimiento. Ingeniare, 18(1), 8-14.

Ross, J., Ross, G., Edvinsson, L., \& Dragonneti, N. (1997). Intellectual Capital: Navigating in the New Business Landscape. London, UK: Macmillan Business.

Saint-Onge, H. (1996). Tacit knowledge the key to the strategic alignment of intellectual capital. Planning Review, 24(2), 10-16.

Sánchez, M. P., Elena, S., \& Castrillo, R. (2009). Intellectual capital dynamics in universities: a reporting model. Journal of Intellectual Capital, 10(2), 307-324. doi: http://dx.doi. org/10.1108/14691930910952687

Stewart, T. A. (1998). La nueva riqueza de las organizaciones: el capital intelectual. Barcelona, España: Granica.

Sukhdev, S., \& Kansal, M. (2011). Voluntary disclosures of intellectual capital: An empirical 
analysis. Journal of Intellectual Capital, 12(2), 301-318.

Sveby, K. E. (1997). The Intangible Assets Monitor. Journal of Human Resource Costing \& Accounting, 2(1), 73-97.

Swart, J. (2006). Intellectual capital: disentangling an enigmatic concept. Journal of Intellectual Capital, 7(2), 136-159.

Toh, M. H., Tang, H. C., \& Choo, A. (2002). Mapping Singapore's knowledge-based economy. Economic Survey of Singapore (Third Quarter), 56-75.
Topete, B. C., y Bustos, F. E. (2007). Propuesta de gestión para las universidades virtuales basada en indicadores de capital intelectual. Innovación Educativa, 7(39), 33-45.

World Bank, International Bank for Reconstruction and Development. (2006). Korea as a knowledge economy: Evolutionary process and lessons learned. Washington, DC., USA: World Bank.

Youndt, M. A., \& Snell, S. A. (2004). Human resource configurations, intellectual capital, and organizational performance. Journal of Managerial Issues, 16(3), 337-360.

¿Cómo citar este artículo? - How to quote this article?

Demuner-Flores, M. del R., Nava-Rogel, R.M., y Mercado-Salgado, P. (2016, junio). Alternativas de gestión del capital estructural en las instituciones de educación superior. Una propuesta para su evaluación. Cuadernos de Administración, 32(55), 47-58. 